\title{
Estrategias para optimizar el manejo de pacientes con faringitis
}

\section{Objetivo}

Evaluar el impacto de diferentes estrategias según distintas guías de práctica clínica sobre el diagnóstico y tratamiento de la faringitis por estreptococo beta hemolítico del grupo A (SBHGA) en adultos y niños.

\section{Diseño}

Estudio de corte transversal para valorar las características operativas de diferentes estrategias diagnósticas en el abordaje clínico de la faringitis aguda.

\section{Lugar}

Un centro de medicina familiar en Alberta, Canadá.

\section{Pacientes}

Se incluyeron 787 pacientes de 3 a 69 años con dolor de garganta entre 1999 y 2002. La información fue aplicada a un puntaje clínico (Tabla 1) para predecir la probabilidad de faringitis por SBHGA. Para su inclusión, debían presentar un puntaje de 2 ó más (ya que con un puntaje de 0 o 1 la probabilidad de SBHGA es menor al 10 $\%)$.

Tabla 1. Puntaje de Centor modificado

\begin{tabular}{l|c}
\multicolumn{1}{c|}{ Griterio } & Puntaje \\
Temperatura > 38 & 1 \\
\hline Ausencia de tos & 1 \\
\hline Inflamación, dolor de ganglios cervicales & 1 \\
\hline Exudados o inflamación amigdalina & 1 \\
\hline Edad & 1 \\
\hline 3-14 años & 0 \\
\hline $\mathbf{1 5 - 4 4}$ años & -1 \\
\hline $\mathbf{4 5}$ años 0 mas &
\end{tabular}

Estrategias diagnostico-terapéuticas comparadas:

1) Cultivar a todos los niños y adultos con dolor de garganta y tratar sólo a los positivos (test de referencia*); 2) Niños: test rápido a todos y tratar a los positivos; cultivar a los que tenían test rápido negativo, y tratar a los positivos. Adultos: test rápido a todos, tratar a los positivos, sin confirmar con cultivo a los negativos; 3 ) En niños: ídem estrategia 2. Adultos: test rápido a todos con Centor 2 o 3 y tratar a los positivos; tratar a todos con Centor 4 o más; 4) Niños: ídem estrategia 2. Adultos: Sin test, tratar a los Centor 3 o $4 ; 5)$ Cultivo en todos los niños y adultos con Centor 2 o 3 y tratar a los cultivos positivos. Tratar a los Centor 4 o más; 6 ) Test rápido a todos los niños y adultos, y tratar los resultados positivos, sin confirmación con cultivo si el test es negativo.

\section{Medición de Resultados Principales}

Sensibilidad $^{*}$ y Especificidad ${ }^{*}$ de cada estrategia para identificar pacientes con faringitis por SBHGA, porcentaje de pacientes en los cuales se prescribiría antibióticos, porcentaje de antibióticos prescriptos innecesariamente en pacientes con cultivo negativo.

\section{Resultados Principales}

De 918 pacientes evaluados, del $86 \%$ se obtuvieron datos completos. La prevalencia de SBHGA en niños y adultos fue del $29 \%$ y $21,9 \%$, respectivamente. En niños, la sensibilidad de las distintas estrategias fue del $100 \%$, excepto la estrategia 6 que fue del 85,8 $\%$. La especificidad fue del $99 \%$ para estrategias 2, 3, 4 y 6 , y del $90 \%$ para estrategia 5 . El uso innecesario de ATB fue del $29 \%$ para la estrategia $5 \mathrm{y}$, para las demás, del $3 \%$. En adultos la sensibilidad fue del $100 \%$ para el cultivo y estrategia 5 , y del $77 \%$ para el resto. La especificidad fue mayor al $96 \%$ para todas, excepto la estrategia 4 que fue del $44 \%$. El uso innecesario de antibióticos fue mayor en la estrategia 4 con el $43 \%$ de utilización innecesaria, mientras que las demás variaron entre 0,6 y 3,3\%.

Tabla 2. Impacto de varias estrategias para el manejo de la faringitis aguda en niños y adultos en la identificación de SBHGA

\begin{tabular}{l|c|c}
$\begin{array}{c}\text { Resultados } \\
\text { Niños }\end{array}$ & Sensibilidad \% (LC 95\%) & Especificidad $\mathbf{\%}$ (LC 95\%) \\
\hline Estrategia 1 & $\mathbf{n = 1 5 5}$ & $\mathbf{n = 2 9 9}$ \\
\hline Estrategia 2 & 100 & 100 \\
\hline Estrategia 3 & 100 & $99,0(97,1-99,8)$ \\
\hline Estrategia 4 & 100 & $99,0(97,1-99,8)$ \\
\hline Estrategia 5 & 100 & $99,0(97,1-99,8)$ \\
\hline Estrategia 6 & 100 & $90,3(86,4-93,4)$ \\
\hline Adultos & 100 & $99,0(97,1-99,8)$ \\
\hline Estrategia 1 & $\mathbf{n = 7 3}$ & $\mathbf{n}=\mathbf{2 6 0}$ \\
\hline Estrategia 2 & 100 & 100 \\
\hline Estrategia 3 & $76,7(65,4-85,9)$ & $99,2(97,2-99,9)$ \\
\hline Estrategia 4 & $78(66,9-86,9)$ & $95,8(92,6-97,9)$ \\
\hline Estrategia 5 & $76,7(65,4-85,8)$ & $43,8(37,7-50,1)$ \\
\hline Estrategia 6 & 100 & $96,5(93,5-98,4)$ \\
\hline
\end{tabular}

\section{Conclusiones}

Realizar test diagnósticos a pacientes adultos seleccionados por el puntaje de Centor, reduciría el uso innecesario de antibióticos. El tratamiento antibiótico solo disminuye en 24 hs la duración de la odinofagia. En niños las estrategias 2, 3, 4 y 5 son viables, ya que la premisa es detectar todas las faringitis por SBHGA, para prevenir la fiebre reumática.

Fuente de Financiamiento: Laboratorio Abbott, Montreal, Canadá. (Fabricantes de los test rápidos utilizados en el ensayo).

\section{Comentario}

Este estudio evalúa diferentes estrategias posibles de manejo de pacientes con faringitis en una población seleccionada ${ }^{1,2}$. Es importante destacar las limitaciones del mismo. En la población adulta no seleccionada, la prevalencia de faringitis por SBHGA es del $10 \%$, mientras que en la población adulta seleccionada para este trabajo fue mayor $(21,9 \%)$ a expensas de descartar aquellos pacientes con puntaje de Centor menor o igual a 1 (se sobreestima la cantidad de pacientes con Centor alto y en consecuencia, el porcentaje de pacientes que recibirán innecesariamente tratamiento antibiótico será mayor). Esto no sería directamente aplicable a la población general, y le resta poder al puntaje de Centor como herramienta diagnóstica, adjudicándole un alto uso innecesario de antibióticos. Debido al mayor uso de recursos de estrategias que testean a todos los pacientes, sería bueno contar con datos de costo-efectividad para evaluar las distintas estrategias. En niños se debe ser muy sensible en el diagnóstico de faringitis por SBHGA para evitar la fiebre reumática.

\section{Conclusiones del comentador}

El puntaje de Centor modificado es una herramienta clínica útil para aplicar en adultos y discriminar aquellos casos que pudieran beneficiarse del tratamiento antibiótico. En niños, es recomendable realizar un test diagnóstico con el fin de tratar solo a aquellos en los que el test resulte positivo. La ventana para comenzar el tratamiento antibiótico, sin perder efectividad para prevenir la fiebre reumática, es de 9 días. Si no se dispone de test diagnóstico. Está indicado el tratamiento antibiótico de todos los niños con Centor mayor a uno.

Dr. Tomas Smith [ Unidad de Medicina Familiar y Preventiva del Hospital Italiano de Buenos Aires ]

* ver glosario

Smith T. Estrategias para optimizar el manejo de pacientes con faringitis. Evid. actual. pract. ambul. 2005;8:12. Comentado de: Empirical validation of Guidelines for the Management of Pharyngitis in Children and Adults. Mclsaac W; Kellner J; Aufricht P y col. JAMA 2004; 291: 1587-1595. PMID: 15069046

Referencias

1.Bisno AL, Gerber MA. Practice guidelines for the diagnosis and management of group A streptococcal pharyngitis. Clin Infect Dis. 2002;35:113-125

2.Mclsaac WJ, White D. A clinical score to reduce unnecessary antibiotic use in patients with sore throat. CMAJ. 1998; 158:75-83. 